\title{
Infant home respiratory monitoring using pulse oximetry
}

Paul Seddon ${ }^{1}$, Sonia Sobowiec-Kouman ${ }^{1}$ and David Wertheim ${ }^{2}$.

${ }^{1}$ Respiratory Care, Royal Alexandra Children's Hospital, Brighton, UK and ${ }^{2}$ Faculty of Science, Engineering and Computing, Kingston University, Surrey, UK.

Corresponding author:

Dr Paul Seddon

Respiratory Care

Royal Alexandra Children's Hospital

Eastern Road

Brighton BN2 5BE

Email: seddop@gmail.com

Tel: +44 (0)1273696955

Fax: +44 (0)1273 523141

Keywords: respiratory rate, infant, pulse oximetry

Title: 7 words

Abstract: 150 words

Body (excluding title page, abstract, tables, figures, and references): 1199 words 


\begin{abstract}
Respiratory rate $(R R)$ is a valuable early marker of illness in vulnerable infants, but current monitoring methods are unsuitable for sustained home use. We have demonstrated accurate measurement of RR from brief recordings of pulse oximeter plethysmogram (pleth) trace in term neonates in hospital. This study assessed the feasibility of this method in preterm infants during overnight recordings in the home. We collected simultaneous overnight $\mathrm{SpO}_{2}$, pleth and RIP on 24 preterm infants in the home. RR from pleth analysis was compared to RR from RIP bands; pleth quality was assessed by presence of visible artefact. Median (range) RR from RIP and pleth were not significantly different at 42 (25-65) and 42 (25-64) breaths/minute. Median (range) \% of epochs rejected due to artefact was 20 (8-75) for pleth and 10 (3-53) for RIP. Our results suggest that home respiratory rate monitoring by pulse oximeter pleth signal is accurate and feasible.
\end{abstract}

\title{
What is known about this topic
}

- Respiratory rate is a key vital sign in young children, and tachypnoea is an important early marker of a range of diseases

- Existing methods for recording respiratory rate, such as respiratory inductive plethysmography or nasal thermistor, are problematic for sustained home use

- The plethysmogram recorded by pulse oximeters contains respiratory data which can be extracted by appropriate signal processing

\section{What this study adds}

- Prolonged respiratory rate monitoring of preterm infants in the home is feasible using a pulse oximeter, with no additional sensors on the infant

- This technique has the potential to allow early identification of deterioration in selected vulnerable infants and young children 


\section{Introduction}

Elevated respiratory rate can be an early indicator of illness in infants and children. To compensate for impaired gas exchange, infants and young children increase minute volume predominantly by increasing respiratory rate to maintain blood oxygenation. Tachypnoea in infants is an early and consistent marker of a number of acute conditions including infection ${ }^{1}$, and can also be the presenting sign in a wide variety of congenital respiratory, cardiac and neurological disorders ${ }^{2}$. Thus respiratory rate is a key measurement for sick and vulnerable infants and children ${ }^{12}$, and monitoring it could help in early identification and treatment of disease. However, at present monitoring respiration requires the use of sensors such as effort bands or nasal thermistor, which are problematic for regular clinical use.

Infants born preterm, especially those who develop chronic lung disease of prematurity, remain vulnerable after discharge from hospital, and are an example of a group in whom respiratory rate monitoring could be helpful. Pulse oximetry is already widely used in these infants for monitoring of oxygen saturation $\left(\mathrm{SpO}_{2}\right)$ and heart rate in the home, and placement of a pulse oximeter sensor on a limb is well tolerated by infants.

Pulse oximeters produce a plethysmogram (pleth) trace reflecting the instantaneous volume of blood in the extremity under the probe, and this pleth trace is affected by changes in respiratory effort ${ }^{3}$. Low pass filtering can be used to remove the dominant heart rate component and yield a trace containing only the lower frequency components. Using this method we have previously derived respiratory data from short segments of the pulse oximeter pleth trace in healthy newborn infants ${ }^{3}$ and for longer periods in infants undergoing investigation in hospital for sleep-disordered breathing ${ }^{4}$. The feasibility of this technique for prolonged infant home recordings has not previously been established.

\section{Aim}

The aim of this study was to examine if respiratory rate can be derived from home pleth recordings in infants born prematurely. Specifically, we wished to establish, in this clinical setting, a) whether respiratory rate measurement is accurate with reference to a standard method (respiratory inductive plethysmography) and b) whether respiratory rate can be monitored for a useful proportion of the duration of recordings.

\section{Method}

The study was approved by the NRES Ethics Committee West Midlands - Edgbaston. A Nonin pulse oximeter module and sensor (Nonin Medical Inc., USA) was used to record a continuous pleth trace, oxygen saturation and pulse rate in two groups of infants born at less than 37 weeks gestation. One group were healthy preterm infants with no current respiratory disease and the second group were preterm infants who subsequently developed chronic lung disease of prematurity (CLD), defined as persisting oxygen requirement at 28 days of age. Simultaneously, respiratory movements were recorded using thoracic and abdominal respiratory inductive plethysmography (RIP) bands, and both 
RIP and pulse oximetry data were recorded on a SOMNOscreen Plus multichannel recorder (SOMNOmedics GmbH., Germany) .

Parents applied equipment in the home after instruction in hospital. The pulse oximeter probe was placed on the foot under clothing and bedding to minimise possible movement related artefact, and recordings were made during natural overnight sleep.

Pleth traces were low pass filtered (LPF) to extract respiratory data employing a similar technique to that previously described ${ }^{34}$ using software we developed with MATLAB (The MathWorks Inc., Natick, $M A, U S A)$. The LPF cutoff frequency was at half the $90^{\text {th }}$ percentile pulse rate calculated in sequential epochs of length 2 minutes; the filter characteristics give a steep roll-off to remove the pulse rate component. The data recorded were visually reviewed using a signal viewer in 1 minute sections. Respiratory rate was computed in sections with little or no artefact of the LPF pleth and RIP traces. In all but one case the LPF pleth trace was compared with the abdominal RIP band; in one case the abdominal band did not record so the thoracic band was used for comparison.

In order to evaluate how sensitive the two techniques are to artefact, the first hour after the traces had settled were assessed in the 21 analysed recordings. The proportions of 60 -second epochs with over 10 seconds of artefact for the LPF pleth data and separately for the RIP band data were evaluated by visual examination.

Data were tested for consistency with a Normal distribution using the Ryan-Joiner test in Minitab v16 (Minitab Inc., USA) and analysed using parametric or non-parametric tests as appropriate.

\section{Results}

Home recordings were analysed from 21 preterm born infants. Three other recordings were not analysed because of technical problems: no RIP band trace in one, no pulse rate and $\mathrm{SpO}_{2}$ data in another and no pleth trace in the third. Nine of the 21 analysed recordings were from infants with CLD; seven of the nine CLD infants were receiving supplemental oxygen at the time of the home recording. Twelve of the 21 recordings were from infants who were born prematurely but had no respiratory or other medical compromise (healthy preterm group).

Table 1 summarises the descriptive data for all infants and the respiratory rate measurements using the two methods. As expected, the gestation and weight at birth of the healthy preterm infants was greater than the CLD preterm infants. However, at the time of recording the postnatal ages and weights were comparable. Seven of the nine CLD infants were receiving supplemental oxygen at the time of the home recording.

Overall the respiratory rate from bands was median (range) 42 (25 to 65) breaths / minute. Respiratory rate and pattern from LPF pleth trace was similar to bands (Figure 1 shows 2 examples) with median (range) 42 (25 to 64 ) breaths /minute (Table 1). The median difference RIP-pleth difference was 0 and range -1.75 to 6.5 breaths /minute (figure 1 lower graph), the BA plot indicating no difference in accuracy at higher rates. 
For recordings on 21 infants, the median (range) \% of epochs rejected due to artefact was 20 (8 to 75 ) for pleth and 10 (3 to 53) for RIP. Those recordings with lower levels of band artefact tended to have lower pleth artefact; there was a relation between the percentage of epochs rejected for pleth and RIP band data (Spearman's rank correlation coefficient $\rho=0.88, p<0.001$ ).

\section{Discussion}

Our results demonstrate that respiratory rate can be derived accurately from good quality pleth home recordings in infants. Although more epochs were affected by artefact on the pleth than on band recordings, overall $80 \%$ of one-minute pleth epochs had no or minimal artefact. This method may thus enable near-continuous additional respiratory rate and rhythm to be derived using standard oximetry sensors.

Visual counting of respiration rate is problematic: the precise method used, duration of counting and state of arousal all influence the value obtained ${ }^{5}$. In selected vulnerable infants and young children, respiratory rate data and trends recorded in the home, using the technique we have developed, could allow early identification of tachypnoea and of pathological respiratory patterns ${ }^{4}$. In oxygendependent infants already undergoing intermittent overnight assessment of saturation and heart rate, respiratory rate would add additional clinical value and may flag up infants not yet ready to "wean". In the future, continuous monitoring home monitoring of these parameters in very high risk infants is a possibility.

Acknowledgements

This research was commissioned by the National Institute for Health Research (NIHR) - RfPB grant PB-PG-0610-22433. The views expressed are those of the authors and not necessarily those of the NHS, the NIHR or the Department of Health.

\section{References}

1. Young Infants Clinical Signs Study Group. Clinical signs that predict severe illness in children under age 2 months: a multicentre study. Lancet 2008;371(9607):135-42.

2. Balfour-Lynn IM, Rigby ML. Tachypnoea in a well baby: what to do next? Arch Dis Child 2015;100(8):722-7.

3. Wertheim D, Olden C, Savage E, Seddon P. Extracting respiratory data from pulse oximeter plethysmogram traces in newborn infants. Arch.Dis.Child. 2009;94:F301-F03.

4. Wertheim D, Parsley C, Burgess S, Dakin C, Seddon P. Pulse oximetry plethysmogram analysis could help identify infants with possible apnoeas requiring full investigation. Acta Paediatr 2014;103(5):e222-4.

5. Simoes EA, Roark R, Berman S, Esler LL, Murphy J. Respiratory rate: measurement of variability over time and accuracy at different counting periods. Archives of Disease in Childhood 1991;66(10):1199-203. 
The Corresponding Author has the right to grant on behalf of all authors and does grant on behalf of all authors, an exclusive licence (or non-exclusive licence for UK Crown and US Federal Government employees) on a worldwide basis to the BMJ Publishing Group Ltd, and its Licensees to permit this article (if accepted) to be published in Archives of Disease in Childhood and any other BMJPGL products and to exploit all subsidiary rights, as set out in our licence.

Competing interest: None declared.

Contributorship statement:

Paul Seddon: : Literature search, study design, data interpretation, writing and review of manuscript Sonia Sobowiec-Kouman : Study design, data collection, writing and review of manuscript David Wertheim: Literature search, study design, data analysis and interpretation, writing and review of manuscript 


\section{Figure Legend}

Figure 1: Upper graph shows examples of two 60 seconds sections from a recording: filtered pleth (top), thoracic band (middle) and abdominal band (bottom trace). The left hand example shows regular respiration whereas the right hand section is an example showing variable respiratory rate and short apnoeas. Lower graph shows Bland-Altman plot of respiratory rate from RIP bands compared with filtered pleth (bands minus LPF pleth vs mean of bands and LPF pleth). The mean (SD) difference is 0.3 (1.56) breaths / min. The orange solid line indicates the mean; dashed blue lines indicate mean $\pm 2 \mathrm{SD}$.

\section{Table legend}

Table 1: Gestation, age and weight details of infants in the study together with respiratory rate derived from pleth traces compared with RIP band recordings. 


\begin{tabular}{|c|c|c|c|}
\hline median (range) & Healthy Preterm & CLD Preterm & All infants \\
\hline Gestation at birth (weeks) & 32 (31 to 34$)$ & 26 (25 to 29$)$ & 31 (25 to 34$)$ \\
\hline Birth weight (g) & 1750 (1380 to 2275$)$ & 867 (557 to 1157 ) & 1405 (557 to 2275 ) \\
\hline Gender & $8 \mathrm{M} \quad 4 \mathrm{~F}$ & $4 \mathrm{M} 5 \mathrm{~F}$ & $12 \mathrm{M} 9 \mathrm{~F}$ \\
\hline Age at recording (months) & $3(1$ to 6$)$ & 5.5 (1.5 to 6.5$)$ & $5(1$ to 6.5$)$ \\
\hline Weight at recording (g) & 4600 (2390 to 6500$)$ & 5260 (3950 to 6350) & 4800 (2390 to 6500$)$ \\
\hline $\begin{array}{l}\text { RIP band respiration rate } \\
\text { /minute }\end{array}$ & 48.5 (31.5 to 65$)$ & $36(24.5$ to 51$)$ & $42(24.5$ to 65$)$ \\
\hline $\begin{array}{l}\text { LPF pleth respiration rate } \\
\text { /minute }\end{array}$ & 47 (31.75 to 64$)$ & 36.5 (24.5 to 50.5$)$ & 42 (24.5 to 64$)$ \\
\hline $\begin{array}{l}\text { Difference } \\
\text { RIP band - LPF pleth / } \mathrm{min}\end{array}$ & $0(-1.75$ to 6.5$)$ & $0(-1.5$ to 0.5$)$ & $0(-1.75$ to 6.5$)$ \\
\hline
\end{tabular}

Table 1 